Itinéraires Itinéraires

Littérature, textes, cultures

\title{
Récits du corps au Japon
}

\section{Marc Kober}

\section{OpenEdition}

\section{Journals}

Édition électronique

URL : http://journals.openedition.org/itineraires/1487

DOI : $10.4000 /$ itineraires. 1487

ISSN : 2427-920X

Éditeur

Pléiade

Édition imprimée

Date de publication : 1 novembre 2011

Pagination : 19-32

ISBN : 978-2-296-55720-8

ISSN : $2100-1340$

Référence électronique

Marc Kober, «Récits du corps au Japon », Itinéraires [En ligne], 2011-3 | 2011, mis en ligne le 01 novembre 2011, consulté le 19 avril 2019. URL : http://journals.openedition.org/itineraires/1487 ; DOI : 10.4000/itineraires. 1487

\section{(c) (i) () $९$}

Itinéraires est mis à disposition selon les termes de la licence Creative Commons Attribution - Pas d'Utilisation Commerciale - Pas de Modification 4.0 International. 


\title{
Récits du corps au Japon
}

\begin{abstract}
The body matter can be transferred to Japan history as "sakoku" (鎖国), a very long isolated country. Japanese body would build up through symbolic stories and views describing natives' nature and culture in contrast with foreigner's body. Is the body omnipresence in Japanese stories significant for all that? The narrative construction of the Japanese body goes through a genealogy, a whole inherited native cultural mediations before the great confrontation with the West. The literature would confirm not so the existence of a body image as a relation to others opening up a specific body field. The story of an improbable "Japanese body", tale for inner and outer use, and as such is a party to a western contemporary view.
\end{abstract}

Keywords : story, body, Japan, discourse, western world Mots clés : récit, corps, Japon, discours, Occident

Pour essayer de mieux comprendre ce que représente le corps pour la société japonaise, et pour nous autres Occidentaux, il faut se rappeler que, comme toute société, celle-ci se sert de ce puissant symbole pour parler de ses fantasmes. En tout cas, cette hypothèse mérite d'être prise en compte ${ }^{1}$. Si l'on suit la chronologie de ce symbole à part, il faut également aborder une diversité de discours très grande, et qui a varié dans le temps ${ }^{2}$. La question du corps entre en résonance avec l'histoire du Japon, qui s'est dans un premier temps défini comme isolé, pays aux ports fermés par des chaînes (sakoku, 鎖国) : une fermeture toute relative qui serait magnifiée avec nationalisme en pureté ethnique. À partir de l'ouverture de l'ère Meiji, début d'une occidentalisation voulue, et en partie maîtrisée, deux types de corps se rencontrent et s'évaluent. Les Européens, puis les Américains, vont étudier le Japon, jusqu'à traquer une différence anatomique, un corps insulaire, et même un caractère national déterminé par un espace physique hors du commun. L'ouverture initiale va se renouveler de façon radicale

1. Michel Bernard, Le Corps, Paris, Seuil, coll. « Points. Essais », 1995, p. 134.

2. Ibid. 
avec l'occupation américaine après la défaite de 1945. La présence américaine précipite une évolution radicale des mentalités et des perceptions, évolution toutefois déjà largement engagée depuis des siècles. Dans les deux cas, les Japonais considèrent leurs corps comme indignes, et la cause d'une défaite, ou d'une infériorité technologique. Les récits de l'après-guerre disent souvent ce sentiment d'infériorité, et le désir (parfois) d'une transformation radicale du corps pour le rendre puissant, beau, hyperactif, ou bien pour l'effacer. L'omniprésence du corps dans les récits japonais est-elle pour autant significative? Serait-elle liée aux récits premiers qui affirment la naissance d'un peuple dont les îles sont d'origine organique, un corps insulaire habité? Ou bien s'agit-il d'une reconstruction fantasmatique d'un corps de synthèse, constitué d'emprunts? Les remarques suivantes auront pour objet le récit d'un improbable « corps japonais », fable à usage interne autant qu'externe, et à ce titre partie prenante du discours contemporain.

\section{Récits du corps au Japon}

La première question serait de savoir si le corps est l'objet d'un récit particulier au Japon, et enfin de savoir qui est responsable de ce récit. En réalité, il s'agit d'un récit composite né d'influences variées, et parfois contradictoires, et qui appartient de plein droit à une culture en constante métamorphose, plutôt qu'à une culture pure et abstraite, qui n'existe que dans la perspective d'une utilisation idéologique du corps. Dans ce cas, les textes à analyser seraient ceux qui relèvent du japonisme, considéré comme une branche du discours orientaliste, mais aussi tous ceux qui tendraient vers un auto-orientalisme autochtone favorisé par la diffusion et la traduction, à usage interne et externe. Le cas de l'œuvre de Mishima est exemplaire de cette part essentielle donnée au corps dans le récit, non moins que celle de Kawabata, ou celle de Tanizaki, auteurs les plus lus et les plus traduits. La seconde question serait de savoir s'il existe un récit japonais du corps. Mais quel récit, sinon d'une part, celui qui a trait au corps imaginaire, récit esthétique, culturel, littéraire, qui l'idéaliserait ou le dévaloriserait, de l'autre, celui qui a trait au corps objectif, comme celui d'un anatomiste, avec des présupposés raciaux, qui isoleraient tel ou tel aspect comme typique $^{3}$. Ces récits du «corps japonais » sont majoritairement extérieurs, pour tout dire marqués par le regard européen. On trouverait là une approche néo-coloniale, et une curiosité condescendante. Ainsi, le corps japonais, vu par un auteur français masculin, est-il réductible à un ensemble de traits qui renvoient essentiellement au corps féminin, objet d'une libération sexuelle globale, ou produit d'une civilisation considérée à tort ou à raison comme ouverte à toutes les formes d'érotisme, ce que réfléchit à sa 
manière la littérature. De nombreux romans français abordent la sexualité de la jeune femme japonaise même si, bien entendu, les romanciers français savent traiter d'autres sujets. L'essai d'une Française ${ }^{4}$ synthétise agréablement un trait culturel japonais présenté comme dominant, même s'il semble fasciner surtout les Français et les Occidentaux, et peut-être, en retour, les Japonais eux-mêmes ${ }^{5}$. Le corps est un " produit », et il est consommé, dans un système général d'économie sexuelle décrit par Baudrillard. Il entre dans un circuit fermé, et répond à des besoins extérieurs à lui-même. Dans une perspective en partie anatomique, mais surtout esthétique, le corps japonais se spécialise et se condense en certains traits jugés typiques, dont le contraire serait un type nordique, jugé particulièrement éloigné. D'où l'oxymore absolue et pourtant réalisée artificiellement de la « geisha blonde ${ }^{6}$ », ou du Japonais aux yeux bleus. Enfin, se construit par métonymie ou par synecdoque - cheveux noirs, peau blanche colorée de l'intérieur par une ombre, nuque maquillée, paupière, œil bridé, longueur du buste, jambes courtes, légèreté, morphologie plate, nez court, visage rond et sans relief, etc. - un « corps japonais » fantasmatique.

La grande majorité des récits français (est-ce parce qu'ils sont écrits par des hommes?) se confrontent au corps japonais dans sa nature jeune et féminine. Le corps est chargé d'exprimer un sujet, une identité individuelle et collective, qui serait la femme japonaise. Corps produit s'il en est par le regard des auteurs (ou des artistes). Rares sont les héros japonais décrits dans leur nature corporelle. Tout aussi rares les corps vieux, ou marqués par le temps. Cette stéréotypie, qui identifie une culture à un corps de jeune femme, est-elle pour autant propre au Japon? Ou bien s'agit-il d'un trait de représentation universel? Une idéalisation collective? Le fait que la variété des morphologies soit réduite à un seul type est certainement un effet de la centralisation culturelle et politique impériale, avec Kyôto comme capitale, puis Edo, devenu ensuite Tôkyô. L'homogénéisation des traits physiques ou de la perception du corps vient contredire le jugement populaire, qui identifie tel type de beauté féminine à telle ville. Ainsi, Kawabata Yasunari serait par excellence l'écrivain de la beauté de Kyôto; Tanizaki Junichirô, celui de la beauté de l'époque Edo, avec certains décrochements vers la culture du

4. Agnès Giard, L'Imaginaire érotique au Japon, Paris, Albin Michel, 2007.

5. Dans le style «flamboyant", nous pourrions citer Jean Pérol, Le Soleil se couche à Nippori, Paris, La Différence, 2007; Laurent Peireire, Le Journal de Kikuko, Seyssel, Champ Vallon, 2005, et Patrick Grainville, Le Baiser de la pieuvre, Paris, Seuil, 2010. D'une manière réaliste, pour le traitement de la relation hommes français-Japonaises : Régis Arnaud, Tôkyô, c'est fini, Lyon, Valat, 1999, ou Frédéric Boilet, Tôkyô est mon jardin [remarquable roman en bande dessinée], Paris, Casterman, 1997. Enfin, dans une approche uniquement «érotique » et «trash », Antoine Misseau, Tokyo Rhapsodie, Paris, La Musardine, 2008. Tous ces romans tournent autour de la sexualité de la jeune femme japonaise. Et ils sont loin d'être les seuls (citons encore Romain Slocombe, ou Roland Jaccard).

6. Voir le roman grand public de Jina Bacarr, Blonde Geisha, Paris, Harlequin, 2009. Paru sous le titre The Blonde Geisha. 
Kansai. Bien des différences sont remarquées par les Japonais eux-mêmes, et nommées par des expressions imagées, tout comme la couleur du miso $^{7}$ diffère du Nord au Sud. Le brassage géographique des identités, qui fait que les clients d'une même nomiya à Tôkyô peuvent se vanter d'appartenir à des régions toutes différentes, et la concentration des corps en quelques lieux à très haute densité (plaine du Kantô, Kansai), doivent-ils pour autant aboutir à cet effet de massification, et à la tyrannie du nombre? Le corps japonais est perçu comme multiple et uni - effet de confusion, d'égarement, par la similitude apparente. Un corps identique (ou démultiplié), dont la photographie peut donner une idée ${ }^{8}$, ou la réflexion de l'essayiste, comme le fit Roland Barthes avec son Empire des Signes. À la limite, il est confondu avec le corps chinois, ou asiatique, dans une proximité qui égare. Effet de distance, ou perte de discernement? Vieux souvenir du « péril jaune », quand ce corps n'est pas purement et simplement privé de toute humanité...

Le récit du « corps japonais » est donc fondamentalement celui d'un objet extérieur qui ne recoupe que partiellement la réalité physique. Pour autant, la version japonaise du même récit vient renforcer son essentialisation. Par de subtiles assertions (celles du voyageur, mais ici dans un voyage interne, au cœur de sa propre culture), Tanizaki dans Éloge de l'ombre, ou Kawabata dans Pays de neige, renforcent l'idée d'un mystère japonais. Et le plaisir du lecteur vient certainement de la résolution partielle de cette énigme corporelle posée à l'orée du texte, et résolue par le déploiement conceptuel et métaphorique de l'ombre ou de la neige.

Les deux polarités de la représentation du corps sont, dans une perspective occidentale, Eros et Thanatos. C'est le corps exultant, au désir libéré qui est privilégié par les Occidentaux. L'exaltation ou le rejet du corps sont deux orientations du récit japonais, anhistoriques, et pourtant liées à l'après-guerre. Un malaise existentiel aurait été anticipé par Akutagawa Ryûnosuke, développé par Ôe Kenzaburô, puis par Yoshimura Akira, par exemple, malaise qui s'exprime par le centrage du récit sur la description du corps. Les modalités de cette utilisation maximale et intensive du corps dans le récit seraient à étudier en termes de saturation de corps inertes, morts, ou endormis (Les Belles Endormies), captifs, dociles ou rebelles. Les récits japonais relatent souvent des expériences corporelles extrêmes.

\section{Le récit indirect d'une nature autochtone}

Un dialogue s'engage avec le corps des autres, ou avec son propre corps (Mishima Yukio dans Confession d'un masque, et dans Le Soleil et

7. Pâte de soja fermentée d'usage quasi quotidien dans la soupe. La couleur varie du très clair au très foncé suivant les régions.

8. Le photographe Araki a parcouru le Japon pour photographier les habitants de villages entiers, ou de quartiers. Ces portraits sont agencés ensuite en mosaïques page après page, dans une longue série d'albums et dans certaines expositions. 
l'Acier). Les corps des vivants se confrontent au corps des morts. Les corps se parlent, disent le mal-être (ou l'épanouissement), comme s'ils étaient des personnages à part entière du récit. Ces corps sont-ils dépeints d'une manière nouvelle et originale par rapport à d'autres civilisations? C'est une question comparatiste complexe. Étiemble l'a posée pour la dimension érotique du corps ${ }^{9}$. Elle réside certainement dans un passé ethnique. Le regard étranger adhère fortement à cette idée : aux origines du Japon, dans le passé, se trouve la vraie originalité japonaise, y compris dans la relation au corps, dans sa représentation. Le principe de base est d'exalter cette différence (érotique) à travers les cultures. Le bouddhisme, aspect frappant de la culture japonaise, est pourtant considéré comme moins significatif que le shinto. Est-ce en raison de la moins grande variété des corps représentés, et de leur dimension asexuée? À l'origine du Japon, on trouverait la multiplicité des corps et leur invention à profusion : « entre huit cents et huit cent mille myriades de divinités polymorphes nommées kami », organisés en une " véritable anarchie de l'imaginaire ${ }^{10} »$.

Le Japon a tenu à conserver le double récit des mythes et des légendes, avec le Kojiki, et le Nihon-shoki. Dans les mythes initiaux de la conception du monde, on rencontre très vite un couple initial, Izanami et Izanagi, qui donne naissance à des îles, puis à l'archipel tout entier, en remuant le magma de l'univers avec une lance. Les gouttes deviennent des îles. Ils observent leurs organes sexuels, dansent et s'unissent avec beaucoup de naturel. La suite de ce mythe initial est tout aussi importante. Après l'énoncé d'une sexualité innocente, sans pudeur, vient dans une seconde étape, la souffrance liée à l'enfantement (Izanami brûlée par le dieu du feu), et l'impureté de la chair (le royaume des morts, le Yomi; le corps brûlé et décomposé). La mort est liée à un hymne érotique, et la souillure appelle la purification.

Ces éléments colorent une certaine manière de concevoir le corps au Japon, et de le raconter en littérature, jusqu'à l'expression fantastique. Un autre passage connu des mythes initiaux est celui où la déesse Ame-no-Uzume danse pour faire sortir Amaterasu, la déesse du soleil. Mythe sexuel (elle dévoile ses organes), cette danse transforme la sexualité en spectacle, en un exhibitionnisme religieux. En apparence, c'est une scène de théâtre panique, qui exorcise une angoisse collective. Il s'agit tout autant d'une domination de la nature par l'utilisation rusée des pouvoirs sexuels féminins. L'association entre art et séduction érotique aura une longue descendance, et hantera bien des récits. Le dédain pour le nu féminin, et l'horreur éprouvée devant l'objet naturel, se retrouvent dans la littérature de l'époque Heian, avec Murasaki

9. Étiemble, L'Érotisme et l'Amour [1987], Paris, Le Livre de poche, coll. « Biblio Essais », 1990. Rien de neuf selon lui en Chine, ou au Japon : «L'homme est partout le même devant sa chair. Quand sa religion ne le brime pas trop, partout il s'invente les mêmes plaisirs ingénieux. » Toutefois, il rajoute : « Mais dans les intentions, les canons esthétiques, quelle heureuse variété! »(p. 10). Le débat reste donc largement ouvert.

10. Théo Lesoualc'h, Érotique du Japon, Paris, Pauvert, 1968, p. 13. 
Shikibu qui rappelle combien était insoutenable la vision du corps dénudé. Par la suite, les premiers nus peints à la manière européenne firent scandale.

Pourtant, est-ce le signe d'une irrémédiable occidentalisation, le Japon se tourne résolument vers l'anatomie, et repousse les représentations grotesques ou monstrueuses. Le Japon entre dans l'âge rationnel, et rencontre d'autres corps, ceux des étrangers, mais aussi leur façon de comprendre le corps humain. Après leurs contacts avec le monde chinois, c'est par les « études hollandaises » (rangaku), du XVII ${ }^{\mathrm{e}}$ siècle jusqu'à la fin de l'ère Edo, que les Japonais s'ouvrent au monde extérieur. Le corps est désormais objet de science. La réalité corporelle se représente anatomiquement. Entre la fin $\mathrm{du} \mathrm{XIX}^{\mathrm{e}}$ siècle et le début du $\mathrm{XX}^{\mathrm{e}}$ siècle se déroulent les principales expositions hygiénistes. La question de la santé touche désormais le « corps national ». L'utilisation habile du sexe comme spectacle se métamorphose pour les besoins de l'explication scientifique. Ces expositions furent très suivies au début du $\mathrm{XX}^{\mathrm{e}}$ siècle. Elles sont révélatrices de l'intérêt de la foule pour les modèles anatomiques proposés. L'éducation médicale des masses se développe ainsi, et le regard que les Japonais portent sur leurs corps change progressivement. L'objectif est de changer la société, notamment par l'interdiction de certaines habitudes, ou de pratiques plus marginales, mais appréciées par le peuple, comme « le corps nu, les bains mixtes, les tatouages, ou les luttes entre combattants nus, qu'ils soient hommes ou femmes ${ }^{11} \gg$. En somme, il s'agit d'occidentaliser les corps. On se situe donc aux antipodes de l'origine mythique et de l'originalité supposée du corps japonais. L'ambition de ces expositions est de lutter contre certaines maladies désormais bien connues en Occident (à défaut d'en connaître encore le remède), tuberculose ou syphilis, en exposant des modèles anatomiques, ou en organisant des projections. Dans les années 1920 ou 1930, ces expositions, qui racontaient à leur manière le corps malade et le corps sain, réunirent jusqu'à 300000 visiteurs par ville. Les mannequins représentaient des Japonais. En revanche, lors des premiers spectacles de mannequins réalistes, entrepris par le maître de marionnettes Matsumoto Kisaburô, à partir de 1854, furent montrés des étrangers aux corps difformes, avec de longs bras, de longues jambes, sans ventre, ou avec une poitrine renfoncée. Ces personnages furent imaginés d'après une description littéraire des « barbares étrangers ${ }^{12}$ ». Ce genre de spectacle coïncide avec l'arrivée des bateaux étrangers et la confrontation physique directe. Le lien entre récits traditionnels issus du monde culturel d'Edo et représentation des corps est particulièrement évident dans ces mises en scène qui formaient autant de récits de l'expérience moderne de la rencontre du corps des étrangers, et de la représentation de soi. Kisaburô répondit à plusieurs commandes, avec un art parfait, notamment dans la représentation anatomique d'un aristocrate

11. Ukigaya Sachiyo, « Le Corps donné à voir », EBISU, Études japonaises, $\mathrm{n}^{\circ} 34,2005$, p. 3-37, p. 9 .

12. L'ouvrage s'intitule Wakan sansai zue, ibid., p. 29. 
japonais, ou une représentation de la déesse Kannon. En définitive, le regard porté par le public sur ces mannequins anatomiques était impressionné par la science médicale de leur créateur, mais aussi plein de curiosité pour l'étrangeté et pour l'attrait sexuel, note Ukigaya Sachiyo.

C'est cette même curiosité qui anime les personnages de divers récits, à coloration étrange, ou parfois franchement fantastique que nous allons évoquer à présent. On se trouve alors à l'opposé de la mythologie de l'ineffable différence, plutôt dans un corps à corps avec l'identité japonaise. Ce n'est plus l'époque des premiers étonnements devant les paradoxes de la pudeur et de la nudité racontés par Luís Fróis ${ }^{13}$.

Parmi les premiers récits considérés souvent comme ceux d'une moderne inquiétude figurent ceux d'Akutagawa Ryûnosuke, Le Nez, et Les Kappas, écrits en 1916 et en 1923, année de la mort d'Akutagawa. Le premier se présente comme un apologue qui décrirait le développement d'une forme de folie, et sa résorption, avec pour cause la longueur démesurée d'un nez. Le grand aumônier Zenchi est si affligé par son nez qu'il parvient à le réduire, mais les sarcasmes qu'il voulait éviter, reviennent de plus belle, et il est soulagé de sentir son nez s'allonger de nouveau. Il ne s'agit plus de l'opposition entre le corps de l'étranger (les longs-nez) et le corps japonais, mais d'une forme d'aliénation à soi. Le sujet se dissocie de son corps, ou de son esprit. Il aborde les rivages de la folie. Les kappa sont des créatures apparentées aux batraciens, mais à forme humaine, qui semblent la projection de la folie de l'auteur, ou de son corps malade. L'obsession du corps laid et difforme hante ses dernières œuvres. En effet, l'auteur lui-même subit un affaiblissement physique rapide, et finit par se suicider au cyanure. Ces kappa représentent par ailleurs des êtres hybrides et grotesques, à la manière du Japon pris dans le mouvement violent de la modernisation, ou bien en proie à la montée du militarisme. Plus simplement, les kappa appartiennent au monde des êtres spirituels incarnés en une forme étrange, et doués d'un pouvoir maléfique qu'ils exercent parfois sur les hommes.

À partir de l'expérience de la Seconde Guerre mondiale, Ôe Kenzaburô décrit un univers carnavalesque et subversif, auquel s'appliquerait l'analyse de Mikhaïl Bakhtine sur le "réalisme grotesque ». Le corps est grotesque avant d'être sublime, à l'inverse d'un Mishima qui va directement au sublime. Ôe Kenzaburô se place du côté des victimes, des exclus et des vaincus, comme dans son récit Gibier d'élevage. Loin d'être un récit " sensualiste ", fait uniquement de goûts et de sensations, ou un récit élégant ${ }^{14}$, Gibier d'élevage évoque plutôt un monde primitif, un Japon

13. Voir Traité de Luís Fróis, S.J. (1585) sur les contradictions de mours entre Européens et Japonais, Paris, Éditions Chandeigne, 1993. De nombreux témoignages de l'époque laissent penser à tort aux Japonais que les Européens sont tous roux et sentent le beurre. Le témoignage de Luís Fróis est en revanche beaucoup plus nuancé.

14. Philippe Forest, La Beauté du contresens, Nantes, Cécile Defaut, 2005, p. 180. 
ancien, mais confronté à l'actualité immédiate : l'irruption d'un aviateur noir américain tombé du ciel dans la vie d'un village autarcique. Des corps d'animaux, des ossements, des corps humains sont brûlés dans une carrière à ciel ouvert, faute de crématorium. Ce qui domine, c'est le sentiment écrasant d'une puissance physique redoutable de l'ennemi. Le narrateur dit des soldats étrangers : «L'odeur de leur peau ruisselante de sueur et celle, violemment agressive, de leur corps, flottait sur toute la vallée comme un temps de saison ${ }^{15}$. \ Le fait que le soldat capturé soit un noir le différencie d'un simple ennemi, mais pour d'autres, il est simplement une bête. Son corps gigantesque encombre les esprits, et le caractère inassimilable de sa différence corporelle, est accentué dans le regard intrigué, effrayé, puis séduit des enfants. Il est tantôt considéré comme un gentil animal paisible, tantôt comme une bête inférieure. Chargés de le laver à la fontaine, les enfants admirent sa nudité, comparée à la robe d'un cheval noir. « Il était d'une absolue beauté ${ }^{16}$. » La révélation de sa beauté se fait en même temps que sa virilité considérable. L'histoire se résout en mise à mort, et en une blessure effrayante infligée au corps du narrateur, qui n'était alors qu'un enfant. Et même mort, la carcasse du soldat noir est inassimilable.

La rencontre du corps victorieux de l'ennemi fut traumatisante pour les Japonais. L'écrivain Nosaka Akiyuki le raconte avec un humour grinçant dans Les Algues d'Amérique ${ }^{17}$. Ce récit évoque les incursions de la langue anglaise dans le quotidien japonais, et comment le discours patriotique japonais inclut les arts martiaux, l'art de tenir ses appuis, mais aussi une fatale inégalité physique, une taille décidément trop petite pour lutter à armes égales. Le sentiment de la défaite (chez les hommes) s'ajoute ici à une débâcle virile, une émulation sexuelle, qui fait l'objet d'un roman entier, Les Pornographes. Ce roman relate, sur le mode d'une épopée satirique, le développement encore artisanal de l'industrie du sexe à Osaka, sur toile de fond de pauvreté et d'inhibition intime. Nosaka raconte avec verve l'aventure moderne du corps japonais, à travers l'industrialisation des domaines intimes du sexe et de la mort. En effet, un autre roman est entièrement consacré, non plus à l'univers des « pornographes », mais à celui des entrepreneurs de pompes funèbres ${ }^{18}$. Les deux univers, celui de la petite mort, et celui de la mort proprement dite, se répondent de façon symétrique.

15. Kenzaburô Ôe, « Gibier d'élevage », Dites-nous comment survivre à notre folie, Paris, Gallimard, 1982, p. 27.

16. Ibid., p. 61.

17. Akiyuki Nosaka, La Tombe des lucioles, suivi par Les Algues d'Amérique, Paris, Picquier, 1988.

18. Akiyuki Nosaka, Les Embaumeurs, Arles, Actes Sud, 2001. Pour une analyse approfondie de ce roman, voir Marc Kober, "Marginalité et littérature mortuaire au Japon », Recherches sur l'imaginaire, $\mathrm{n}^{\circ} 30$ : Métiers et marginalité dans la littérature, Angers, Presses de l'Université d'Angers, 2004, p. 55-68. 


\section{Métamorphoses du corps omniprésent}

Si le corps des dieux (Kami) est omniprésent et démultiplié suivant les croyances du shinto, cette surpopulation s'explique aisément dans la mesure où tous les aspects du monde naturel sont potentiellement divins, et peuvent par conséquent s'incarner en différents types de dieux. Ainsi, arbres, rochers, rivières, lacs, mais aussi animaux, constituent des formes divines qu'il suffit de reconnaître, de nommer et de vénérer. La nature est donc particulièrement incarnée. Toutefois, c'est l'univers urbain, d'une densité surprenante qui constitue l'expérience majeure, et parfois presque exclusive des Japonais contemporains, et cette expérience corporelle induit des récits différents.

Si les mythes des origines, en tant qu'il sont des récits archétypaux, ou bien l'expérience historique de la violence et de la douleur (à travers notamment la guerre, à partir de la fin des années 1930, ou l'atomisation par exemple) sont importants dans les choix des écrivains japonais, il est deux domaines où leur imaginaire a été particulièrement productif, et où, par contrecoup, l'influence culturelle du Japon a été maximale dans le monde entier, et nous aimerions développer leur nature : le domaine amoureux, et le domaine fantastique. Là, le raffinement dans l'expression esthétique et littéraire du corps semble particulièrement important ${ }^{19}$.

En ce qui concerne l'univers des corps amoureux, le monde des courtisanes du Yoshiwara (吉原), lieu dit « de la plaine des roseaux », rebaptisé en " plaine du bonheur », a certainement été le cœur littéraire et artistique de l'époque Edo quand l'époque Heian prit pour cœur la ville impériale. Yoshiwara fut un lieu quintessencié qui exprimait plus qu'un autre la culture artistique. Or, ce fut tout d'abord le lieu d'une consommation ritualisée, et d'un embellissement du corps des femmes. Il fut aussi l'occasion d'une ascèse amoureuse, et d'une éducation artistique pour ses habitués. Yoshiwara fut une machine artistique et littéraire, et un lieu de subversion du pouvoir politique. Ouvert en 1618, déplacé au nord de la ville d'Edo, il fut florissant au début du XvIII 'siècle. On le nommait Fuyajo, la « ville sans nuit », et de cette fameuse insomnie naquit une très vaste littérature souvent illustrée. Mais quelle littérature? Elle guide les corps curieux dans Yoshiwara. Ainsi, Le Grand Miroir de la voie de l'amour, rédigé par Fujimoto Kazan ${ }^{20}$, ou Le Guide de l'amour au Yoshiwara, conçu par Hishikawa Moronobu ${ }^{21}$, qui raconte le périple de vingt hommes d'armes et bonzes. Tel lettré possédait au moins 200 livres sur Yoshiwara, et une

19. Voir pour plus de détails, Marc Kober, «Récits de l'intensité - Inscription du corps dans la fiction japonaise », dans Assia Belhabib (dir.), Littérature et Altérité, Rabat, Éditions Okad, 2009, p. 263-282.

20. D'après la référence citée par Christophe Marquet dans son introduction à L'Almanach des maisons vertes, Arles, Picquier, 2008, l'ouvrage fut publié dans Zoku Enseki jisshu, vol. 2, Tôkyô, Kokusho kankôkai, en 1909, mais il fut diffusé auparavant sous forme manuscrite.

21. Ouvrage xylographique de vingt illustrations commentées, Tôkyô, National Diet Library, 1678. 
centaine de documents. Les lettrés et les artistes d'Edo s'intéressaient énormément à ce lieu de prostitution.

Utamaro publie un Almanach des maisons vertes en 1804 qui décrit les rituels annuels et les règles de comportement, le protocole que doit respecter un client. Les sharebon (livres plaisants, dialogues qui reproduisent les tournures utilisées à Yoshiwara) prenaient aussi pour cadre les quartiers de plaisir, pour relancer le rêve et le commerce, écrit Christophe Marquet ${ }^{22}$. Utamaro sera le portraitiste talentueux des courtisanes et de toutes les prostituées. Il publiera trente livres accompagnés de gravures érotiques. Jippensha Ikkû, romancier célèbre pour son récit Sur la route du Tôkaidô, produisit 600 titres, dont des sharebon, et des kyôka, ou épigrammes burlesques. Tous deux sont de parfaits connaisseurs du Yoshiwara, et des artistes inspirés par ce monde flottant (浮世, ukiyo) qui est aussi, à l'envers, monde de souffrance (yo uki). Avec eux, la littérature, l'art et la passion des corps entrent en interaction. Cette littérature, ou ces suites de scènes et de portraits, suggèrent tantôt l'enlacement des corps, plus encore que leur nudité, l'exagération et la minutieuse description des organes dans les shunga (春画, images de printemps), tantôt une stylisation de la fragilité, avec des femmes-fleurs, associées par analogie aux nuages, à un rameau de prunier en fleurs sous la pluie. Des corps de neige qui hantent les almanachs. Edmond de Goncourt dira qu'elles sont « manequinées », corps étirés, déhanchés, ou cambrés à la façon des odalisques d'Ingres ${ }^{23}$. Et la littérature? Pour prendre un seul exemple, l'œuvre de Nagai Kafû, est inspirée par sa propre vie, abritée « à l'ombre des saules », métaphore végétale des courtisanes, alors que c'est déjà l'époque Taishô ${ }^{24}$. Une bonne partie de la littérature japonaise ne saurait se comprendre si l'on n'a pas conscience de cette coïncidence entre création artistique et organisation des rapports amoureux.

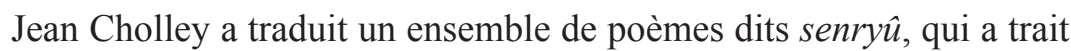
au monde des courtisanes, dont Yoshiwara est la plus haute expression ${ }^{25}$. En dix-sept sons, comme le haïku, se dessine une narration populaire, qui se compte à des milliers d'unités réunies en recueils. C'est le monde des femmes, les pluviers plaintifs, qui sont perdues aux yeux des bouddhistes, à moins de se réincarner dans le corps et l'esprit d'un homme. Le corps est omniprésent dans les senry $\hat{u}$, et tous les usages qui lui sont associés, comme le tatouage du nom du ou des protecteurs, le doigt piqué pour écrire une lettre avec le sang, ou tranché et envoyé à l'amant oublieux. Les différents noms des prostituées sont évoqués, comme les prostituées fusils (teppô) associées au poison du fugu, les yotaka (ou faucons de nuit), les funamanjî, ou brioches sucrées des bateaux. Ces senryû sont essentiellement écrits au

22. Kitagawa Utamaro et Jippensha Ikkû, L'Almanach des maisons vertes, Arles, Picquier, 2008, p. 37.

23. Élizabeth Lemirre, Avant-propos, dans Kitagawa Utamaro et Jippensha Ikkû, op. cit.

24. L'ère impériale qui commence en 1912.

25. Jean Cholley, Courtisanes du Japon, Arles, Picquier, 2001. 
sujet de Yoshiwara. Mis bout à bout, ils forment un magnifique récit. Techniquement, ce sont des haïkus, mais en recherchant le comique d'observation et d'expression. Un autre genre poétique est celui des haïkus érotiques, dont la spécialisation corporelle ne fait pas de doute.

Toujours dans le domaine amoureux, mais cette fois dans la sphère familiale et en dehors de Yoshiwara, certains livres développent une approche naturelle du sexe et de l'union des corps. Il s'agit d'une connaissance du corps de l'autre, de la recherche d'un plaisir naturel, et éprouvé sans terreur. Les makura-e (枕 絵, manuels ou livres de l'oreiller) ont pour but d'expliquer au lecteur (et surtout à la lectrice) comment s'y prendre pour éprouver et donner du plaisir physique ${ }^{26}$. On dit aussi warai-bon (livres qui font rire, ou sourire). Ils contiennent des conseils et des précisions anatomiques. Ce sont des livres en images, mais des gloses et des observations sont incluses dans le dessin, entre les personnages en action, parfois dans la reprise parodique de livres d'enseignement moral. On peut considérer ces ouvrages comme purement spécialisés, et non représentatifs, même si leur production et leur usage furent très importants à une époque donnée. Pour Étiemble, le souci esthétique l'emporte sur l'intention didactique ${ }^{27}$. Il n'est pas impossible que la période considérée, le XVIII ${ }^{\mathrm{e}}$ siècle, coïncide avec la littérature et la peinture libertines qui se développèrent en France à la même époque, dans un esprit tout différent. Mais ce type d'ouvrages a connu une vague continue au Japon, jusqu'au XIX ${ }^{\mathrm{e}}$ siècle. Il est à noter enfin que le souci d'éducation sexuelle explicite n'est pas absent de l'époque actuelle par la présence très répandue de manuels à l'usage des adolescents qui n'ont rien à voir avec leurs équivalents occidentaux par leur minutie.

À propos d'un univers japonais particulièrement ouvert à des représentations fantastiques du corps, nous aimerions développer l'exemple des croyances populaires et folkloriques qui ont trait aux êtres surnaturels, dits yôkai (妖怪) ${ }^{28}$, objets d'une description très riche, iconographique notamment, et qui relèvent parfois du genre fantastique. Un principe essentiel est celui de la métamorphose, ou transformation du corps humain en corps animal, et vice-versa. Le principe de métamorphose est courant dans la Grèce antique, et Ovide a rassemblé les plus célèbres en un recueil qui est l'une des grandes sources de la littérature et de l'art occidental. De ce

26. Jean Cholley, Manuel de l'oreiller, Arles, Piquier, 1997.

27. « On peut toutefois se demander si la part au Japon de la pédagogie dans les traités de l'oreiller l'emporte vraiment sur celle de la beauté et si, du XVII ${ }^{\mathrm{e}}$ au XIX ${ }^{\mathrm{e}}$ siècle, le peintre de shunga ne s'intéresse pas avant tout à la qualité de ses noirs, de ses bleus, à la mise en page » (Étiemble, op. cit., p. 12).

28. Voir Yôkaï : bestiaire du fantastique japonais, Paris, Maison de la culture du Japon, 2006. Dans ce catalogue, Kazuhiko Komatsu insiste notamment sur l'essor des récits illustrés, nés de la conjoncture des images et des mots. Toriyama Sekien (1714-1768) rassembla nombre de yôkai présents dans divers textes et récits populaires, puis il leur donna progressivement des représentations iconographiques. 
point de vue, la mythologie grecque, et les mythes qui se sont développés alors, correspondent souvent à l'imaginaire nippon. Mais les yôkaï sont plus complexes, et leur nature ne se limite pas au simple pouvoir de métamorphose. Du point de vue des Japonais, on parle plus généralement de bakemono (化け物), ou de o-bake (お化け), qui en est l'abréviation. Le verbe bakeru veut dire « se transformer », " se changer en ». Autrement dit, les êtres peuvent se transformer en animaux magiques (le plus connu étant le renard inari), et ceux-ci peuvent prendre une apparence humaine, souvent séduisante. Le maquillage et la métamorphose utilisent un caractère en commun, qui signifie la transformation (化). Ainsi, on dit que les femmes très belles sont en réalité des renardes venus séduire les hommes. Les apparences sont incertaines, et la réalité animale qui se dissimule derrière l'apparence humaine est souvent effrayante. Dans le drame de Dôjôji, Kiyohime est très amoureuse du prêtre Anchin, va l'attendre en vain, et finir par le poursuivre dans son temple. Elle se transforme, sous l'effet de la frustration amoureuse, et en raison de sa très grande passion en un monstrueux serpent, qui finira sous la cloche du temple, la fera fondre, avant d'être anéanti par des formules religieuses efficaces.

Les spectres, ou fantômes, partagent le monde des humains, et entrent rarement en relation avec eux. Les histoires de spectres et de revenants sont très nombreuses, et plaisent particulièrement aux Japonais. Dans ce domaine, l'auteur irlandais et grec, Lafcadio Hearn est un maître. De telles histoires lui furent constamment d'un grand secours pour construire son œuvre $^{29}$. Les kwaidanbanashi sont remplis de morts, de fantômes et de démons (oni). L'idée majeure est celle d'une revanche des fantômes, qui restent puissants et dangereux. Deux mondes coexistent : le monde d'ici et le monde au-delà. Citons notamment les traductions de Yoshimura Akira, La Jeune Fille suppliciée sur une étagère ${ }^{30}$; d'Ikezawa Natsuki, Des os de corail, des yeux de perle, ou Le Faste des morts, d'Ôe. Ces histoires récentes traitent des questions de crémation, de survie des cadavres, et de séparation du mort. À partir de cette réalité sociologique ou religieuse, des légendes et une culture locale se développent.

Un ethnologue en particulier va s'intéresser aux superstitions des gens ordinaires, Yanagita Kunio (1875-1962), et ouvrir le champ du minzokugaku, soit l'étude des coutumes et des traditions populaires du Japon. Il est le premier à vouloir intégrer l'étude des superstitions dans le domaine des études scientifiques.

29. Voir Marc Kober, «L'acte gratuit de Lafcadio Hearn (les légendes et le folklore nippons vus par un écrivain apatride) », dans Véronique Gély, Jean-Marc Moura, Joëlle Prungnaud et Evanghélia Stead (dir.), Les Littératures européennes et les mythologies lointaines, Lille, Édition du Conseil Scientifique de 1'Université Charles-de-Gaulle-Lille3, coll. «UL3, Travaux et recherches », 2006, p. 251-260.

30. La Jeune Fille suppliciée sur une étagère suivi de Le Sourire des pierres, Arles, Actes Sud, 2002. 
Les animaux, ou créatures imaginaires, ont suscité son intérêt à une époque où le Japon s'engageait dans une modernisation méthodique. Cet ethnographe a sauvé les croyances populaires du discrédit jeté par le gouvernement. Il fallait alors adopter à tout prix la science occidentale, et adopter un esprit rationnel en tout. Prenant le relais de Yanagita, le célèbre dessinateur Mizuki Shigeru constitue une « encyclopédie de monstres » (Yôkai zukan). Un autre dessinateur a redécouvert cette culture populaire à travers l'œuvre de ce folkloriste, et lui a donné une interprétation sous forme de manga. Il s'appelle Hanawa Kazuichi. En ce qui concerne Mizuki Shigeru, nous sommes dans l'univers d'un manga-ka, un dessinateur, mais dans son encyclopédie, il prend des notes qui forment le récit des métamorphoses du corps. Ces yôkai sont des vues de l'esprit, mais ils entrent dans la composition parfois de la « grande » littérature : chez Edogawa Rampo, ou Tanizaki. Parmi les yôkai décrits par Mizuki Shigeru, citons comme particulièrement originaux la femme à deux bouches (futakuchi onna), la grande femme haute (taka onna), le batteur de tatamis, le lécheur de plafond, et la face vide (noppera-bô). Le réalisateur Miyazaki Hayao a souvent choisi pour ses films animés de tels animaux, et une multitude d'autres, tels qu'on peut les voir dans Le Voyage de Chihiro, ou dans Mon voisin Totoro. La variété des animaux plus ou moins anthropomorphes, et distincts des yôkai est très importante au Japon.

Pour évoquer les spectres japonais, les Ubume monogatari sont des histoires de femmes enceintes et maltraitées. Les Ubume-kosodate yûrei, sont des femmes mortes qui gardent et nourrissent leur enfant. Nosaka donne un bel exemple des enfants et des mères mortes qui viennent agresser les vivants, dans Le Dessin de sable et l'apparition vengeresse qui mit fin au sortilège $^{31}$. L'obligation pour la mère de nourrir et protéger son enfant n'est pas dissoute par la mort. Le fœus non né ou le bébé non ritualisé d'une mère morte est dangereux, et peut constituer une menace pour les vivants. Il possède une étonnante vigueur et une capacité à courir. Il a une marque, et des cheveux blancs. La crainte par rapport au fœtus dit mizuko (enfant des eaux) est exorcisée par les rites bouddhiques, et stimule un imaginaire angoissant.

Si l'on tente de décrire globalement l'apparence physique d'un fantôme japonais, il se reconnaît à son absence de pieds. On peut dire que le pied qui, chez Tanizaki notamment, est un point d'accroche fétichiste, et une déréalisation de l'être, procurerait, s'il existait, un ancrage dans le réel. L'être dépourvu de pieds appartient à un monde immatériel. Souvent d'apparence féminine, cette créature possède une longue robe, et se déplace de façon immatérielle. Par ailleurs, ses cheveux sont trop longs, ou hirsutes, non coiffés. La chevelure est un indice important pour déterminer si on a affaire à un fantôme. À propos des cheveux, le réalisateur Kobayashi Masaki 
a exploité le recueil Kwaïdan de Lafcadio Hearn pour son film homonyme en quatre parties sorti en 1964. La première partie, "Les Cheveux noirs ", montre comment une femme aimante, mais abandonnée par son mari, réapparaît devant lui, à l'état de spectre, et précisément sous la forme d'une chevelure mobile qui vient l'attaquer, et le rendre fou. Les « femmes fatales » possèdent une longue et belle chevelure noire, rendue brillante par l'huile de camélia, et c'est un des éléments de la beauté à l'apogée de l'ère Heian, aux alentours de l'an 1000, telles qu'on les voit sur les rouleaux illustrés du Genji Monogatari. Mais cette coiffure est bien entretenue, les cheveux propres et lisses. Quand ils deviennent hirsutes et désordonnés, on a affaire à des spectres, ou à de vieilles femmes $(b a b a)$, proches des sorcières. Un autre film montre bien ce type de femmes sauvages : Onibaba, de Kaneto Shindô, sorti également en $1964^{32}$. S'il existe des particularités du récit japonais du corps (et de ses métamorphoses) qui ont été fort goûtées par les Occidentaux, ce sont bien les histoires de revenants, dits yûrei (幽霊), les recueils graphiques et littéraires de yôkai, mais aussi les évocations du monde flottant associé à Yoshiwara.

En conclusion, la littérature japonaise indique une norme physique et un hors-norme tout aussi fortement. Elle propose de lire une diversité considérable de corps et d'apparences, à l'opposé de l'uniformité de surface. Au contraire, une extrême diversité se développe dans l'ordre de l'imaginaire. La culture japonaise, si mouvante et internationalisée à présent, ne se réduit pas à une entité unique. Tout naturellement, dans le cas de la représentation des corps par un récit, se reflètent en lui les soubresauts de l'histoire des derniers siècles, et la succession des mythes dominants. Ce qui veut dire que le corps ainsi raconté, au Japon comme ailleurs, n'est jamais figé dans une posture, ni neutre, mais qu'il est articulé dans un rapport ambigu au pouvoir notamment politique. Tantôt il se tient « à carreau », tantôt il s'effondre et se détraque, comme le corps de l'héroïne de la nouvelle Tatouage de Tanizaki Junichirô. Dans ce cas, il cesse d'être un rouage, et devient le grain de sable qui détraque tous les calculs qui ont pu être fondés sur lui. Et le récit du corps devient alors le récit de la subversion inattendue de toutes nos attentes de lecteurs postjaponistes.

Marc Kober

Université Paris $13-C E N E L$

32. Oni veut dire « démon ». Onibaba, « sorcières ». 